\title{
Disorder classification of the vibrational spectra of modern glasses
}

\author{
Zhiwen Pan, ${ }^{1}$ Omar Benzine, ${ }^{1}$ Shigeki Sawamura $\odot,{ }^{2}$ Rene Limbach,,${ }^{1}$ Akio Koike, ${ }^{2}$ Thomas D. Bennett, ${ }^{3}$ Gerhard Wilde $\odot,{ }^{4}$ \\ Walter Schirmacher, ${ }^{5,6}$ and Lothar Wondraczek (10 1,7,* \\ ${ }^{1}$ Otto Schott Institute of Materials Research, University of Jena, 07743 Jena, Germany \\ ${ }^{2}$ Materials Integration Laboratories, AGC Inc., Yokohama, Kanagawa 230-0045 Japan \\ ${ }^{3}$ Department of Materials Science and Metallurgy, University of Cambridge, Charles Babbage Road, Cambridge, CB3 OFS, United Kingdom \\ ${ }^{4}$ Institute of Materials Physics, University of Münster, 48149 Münster, Germany \\ ${ }^{5}$ Institute of Physics, University of Mainz, 55099 Mainz, Germany \\ ${ }^{6}$ Center for Life Nano Science @ Sapienza, Istituto Italiano di Tecnologia, I-00161 Roma, Italy \\ ${ }^{7}$ Abbe Center of Photonics, University of Jena, 07745 Jena, Germany
}

(Received 16 August 2021; revised 27 September 2021; accepted 29 September 2021; published 22 October 2021)

\begin{abstract}
Using the coherent-potential approximation in heterogeneous-elasticity theory with a log-normal distribution of elastic constants for the description of the Raman spectrum and the temperature dependence of the specific heat, we are able to reconstruct the vibrational density of states and characteristic descriptors of the elastic heterogeneity of a wide range of glassy materials. These descriptors are the nonaffine contribution to the shear modulus, the mean-square fluctuation of the local elasticity, and its correlation length. They enable a physical classification scheme for disorder in modern, industrially relevant glass materials. We apply our procedure to a broad range of real-world glass compositions, including metallic, oxide, chalcogenide, hybrid, and polymer glasses. Universal relationships between the descriptors on the one side, and the height and frequency position of the boson peak, the Poisson ratio and the liquid fragility index on the other side are established.
\end{abstract}

DOI: 10.1103/PhysRevB.104.134106

In contrast to crystalline materials, glasses exhibit aperiodic heterogeneity in local density, chemical composition, and structural rigidity. This complicates the extraction of structure-property correlations and predictive tools for experimental materials design or classification. On the other hand, statistical information may be obtained from the vibrational spectrum of the materials, which can be probed by Raman light scattering and by measuring the temperature dependence of the specific heat. Here we use a mean-field theory which links the vibrational spectrum to the statistics of elastic heterogeneity, to characterize glasses according to their disorder strength and the characteristic correlation length of spatial elastic fluctuations. These descriptors are shown to be related to the elastic properties of the glass and the temperature dependence of the viscosity of the melt ("glass fragility") and may therefore be helpful for designing new materials for specific applications.

The extraordinary spatial homogeneity of glassy materials underlies their many applications, as it leads to, e.g., optical transparency, characteristic fracture patterns, distinct mechanisms of charge, heat and sound transport, or specific solvation behavior. In turn, the ultimate limit of all of these

\footnotetext{
*lothar.wondraczek@uni-jena.de
}

Published by the American Physical Society under the terms of the Creative Commons Attribution 4.0 International license. Further distribution of this work must maintain attribution to the author(s) and the published article's title, journal citation, and DOI. properties lies in the length scale below which material homogeneity is broken. Following the curious observation that the density of a glass is usually lower than that of its crystalline counterpart (when chemical compositions were equivalent) early hypotheses of network formation [1] and excess free volume [2,3] were formulated for different classes of glass. These led to the qualitative paradigm of glass structure, where aperiodic heterogeneity in the atomic packing density, in network rigidity and/or in the distribution of chemical species, constitutes the primary difference to a crystalline lattice. Although the process by which a supercooled liquid acquires the rigid glassy state remains poorly understood $[4,5]$, such types of structural heterogeneity have been related to the heterogeneous dynamics of the liquid [6], liquid fragility [7], and the nonergodicity factor [8].

However, across all types of glass, quantitative descriptors for structural heterogeneity have been elusive, due to the inability to describe glass structure beyond short length scales. As a result of structural disorder, the fundamental tool for understanding crystal properties, namely the lattice symmetry, is not available for glasses. Instead, empirical or semiempirical regression models are frequently used for property predictions. For example, the elasticity of multicomponent glasses is often described through mean-field regression analysis of bond energy density [9,10]. Although useful in their simplicity, such practical approaches ignore localization phenomena and nonaffinity, which are common to disordered materials across the broadest range of length scales [11] and material chemistries [12]. For example, for brittle glasses with strongly covalent bonding, heterogeneous network topology has been 
identified to mediate nanoscale ductility [13] and macroscopic plasticity $[14,15]$.

It turned out that an important property of glasses is the presence of random spatial fluctuations of local elastic constants. It was shown that such heterogeneous elasticity is intimately related to the occurrence of nonaffine displacements [16-20]. In metallic glasses, a parameter denoted flexibility volume was extracted from the spatial distribution of the atomic mean-square displacement as probed by computational simulation [21]. For two- and three-dimensional Lennard-Jones glasses, correlated particle displacements with a correlation length of 20-30 interparticle spacings were found [18]. Similar observations were made in a computergenerated model of amorphous silica [19].

When compared to crystals, the vibrational density of states (VDoS) of glasses exhibits an anomalous excess in the $\mathrm{THz}$ regime, which forms a maximum if the VDoS is divided by the square of the frequency ("boson peak") [22-24]. The VDoS is directly accessible by means of inelastic nuclear [25] and incoherent neutron scattering [26], but only indirectly by inelastic coherent neutron [23,24,27], x-ray [28-30], and Raman [14,31-33] scattering. Further information on the VDoS in the $\mathrm{THz}$ regime can be obtained from the temperature $(T)$ dependence of the specific heat $C(T)[34,35]$, where the boson peak is observed if plotted as $C(T) / T^{3}$. The possible origin of the boson peak has been discussed rather controversely [36-40]. However, many groups nowadays agree that in most cases spatial fluctuations of the local elastic moduli cause this anomaly [41-43], hence, studies of the $\mathrm{THz}$ vibrational anomaly in the VDoS, in principle, provide access to the descriptors of spatial elastic fluctuations.

The boson-peak related anomalies, including a characteristic shoulder in the temperature dependence of the thermal conductivity, can be rather successfully explained and described by heterogeneous-elasticity theory (HET) [44]. Combined with the self-consistent Born approximation (SCBA), a relation of the excess VDoS with the sound attenuation coefficient was established [45] and the results were tested in detail against extensive molecular-dynamics simulations [41]. In the latter study it was also shown that the decrease of the value of the shear modulus with respect to the average one is due to nonaffine elasticity, and that HET accounts for this effect.

As HET describes microscopically the vibrational dynamics of a glass, all spectroscopic data can be expressed in terms of the wave-number-dependent dynamic susceptibilities, which are obtained from the statistics of the fluctuating elastic constants. In particular, the Raman spectra are given as wave-number integrals of these susceptibilities [31]. Therefore, by means of HET, it is possible to unambiguously extract the VDoS from Raman data $[31,33,46]$. This procedure has been demonstrated to be more reliable than using a phenomenological frequency-dependent coupling constant $C(\omega)$, as is widely done in the literature $[14,31,32]$.

However, within SCBA the description of the elastic disorder is restricted to weak and Gaussian fluctuations. As this is not sufficient for the description of a very broad range of disordered materials, HET has been generalized by means of the coherent-potential approximation (CPA) $[36,47]$. Within CPA an arbitrary distribution of local elastic constants can be implemented, and there is no restriction to weak disorder. However, a systematic analysis of a broad class of glasses by means of HET-CPA has not yet been conducted.

We now present the results of a disorder classification of modern glasses using HET-CPA with a log-normal distribution density of spatially fluctuating elastic constants. This variant of HET features specific descriptors of the statistics of the shear modulus, namely the variance, the geometric mean, and the coarse-graining size, which is proportional to the correlation length of the fluctuations. Using the scaling properties of the CPA we obtain a relation between the first two quantities, which reduces the descriptors to the disorder parameter $\sigma^{2}$ and the coarse-graining wave number $k_{e}$. Evaluating the vibrational spectra of a large number of glassy materials we obtain correlations of these descriptors with the boson peak frequency, the boson peak height, the Poisson ratio, and the liquid fragility index. We achieve this goal with experimental input from $\mathrm{THz}$ Raman spectroscopy and low-temperature heat capacity, covering all known classes of glass, from metallic to nonmetallic, chalcogenide, polymeric, and hybrid materials.

\section{THEORY}

\section{A. HET-CPA}

Within HET-CPA the spatially fluctuating local shear moduli $G\left(\mathbf{r}_{i}\right) \equiv G_{i}$ are converted to a macroscopic complex, frequency-dependent modulus $G(z)(z=\omega+i \epsilon, \omega=2 \pi v$ is the angular frequency) by the self-consistent CPA equation

$$
\left\langle\frac{G_{i}-G(z)}{1+\frac{1}{3}\left[G_{i}-G(z)\right] \Lambda\left(k_{e}, z\right)}\right\rangle_{P}=0 .
$$

The (arithmetic) average $\langle\cdots\rangle_{P}$ is performed with a lognormal distribution density

$$
P\left(G_{i}, G_{0}, \sigma\right)=\frac{1}{\sigma \sqrt{2 \pi}} \frac{1}{G_{i}} \exp \left\{-\frac{1}{2 \sigma^{2}}\left[\ln \left(G_{i} / G_{0}\right)\right]^{2}\right\} .
$$

We chose this distribution because (i) it excludes negative values of $G_{i}$, and (ii) for weak disorder $\sigma^{2} \ll 1$ it reduces to a Gaussian distribution ${ }^{1}$ (see Fig. 1), but for $\sigma^{2}$ values around and larger than 1 it accounts for a broad range of non-Gaussian disorder. This latter feature is not shared by other strong-disorder distributions [47].

$G_{0}$ and $\sigma^{2}$ are the first two descriptors of our classification. $G_{0}$ is the geometric mean of the $G_{i}$, also called "typical value," which is for this distribution equal to the median $\sigma^{2}=\ln \left\{1+\operatorname{Var}\left[G_{i}\right] /\left\langle G_{i}\right\rangle_{P}^{2}\right\}$ is the disorder parameter, related in the indicated way to the relative variance. $\Lambda(z)$ is the susceptibility function, which is a linear combination of the longitudinal and transverse local susceptibilities $\chi_{L, T}\left(k_{e}, z\right)$,

$$
\begin{aligned}
\Lambda(z) & =\frac{3}{k_{e}^{3}} \int_{0}^{k_{e}} d k k^{4}\left[\frac{4}{3} \frac{1}{-z^{2}+k^{2} v_{L}^{2}(z)}+2 \frac{1}{-z^{2}+k^{2} v_{T}^{2}(z)}\right] \\
& =\frac{4}{3} \chi_{L}\left(k_{e}, z\right)+2 \chi_{T}\left(k_{e}, z\right) .
\end{aligned}
$$

\footnotetext{
${ }^{1}$ We note that for weak disorder the CPA [47] reduces to the selfconsistent Born approximation (SCBA) [41,44], which is based on Gaussian elastic heterogeneity.
} 


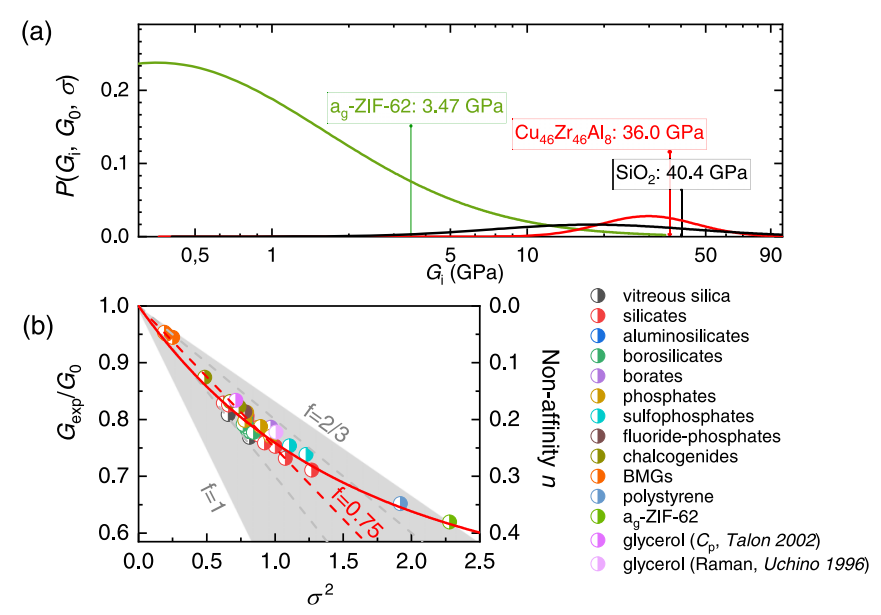

FIG. 1. (a) Distribution $P\left(G_{i}, G_{0}, \sigma\right)$ for some archetypal glasses. The labels indicate the characteristic value of $G_{0}$. (b) Ratio of the shear modulus $G_{\text {expt }}$ and the typical shear modulus $G_{0}$ vs the disorder parameter $\sigma^{2}$ (symbols). Curved red line: $G(0) / G_{0}$ vs $\sigma^{2}$ for $f(\kappa=2)=0.8$ according to the CPA relation (A10). Straight dashed lines: Low-disorder CPA relation (6) for several values of $f$. The shaded area indicates the accessible region within the limits of $f(\kappa)$.

The upper wave-number cutoff $k_{e}$ is the third descriptor of our classification. $k_{e}=\sqrt[3]{2 \pi^{2} / V_{c}}$ is related to the minimum possible coarse-graining volume $V_{c}$ for obtaining statistically independent local shear moduli $G_{i}$ [47]. Therefore, $k_{e}$ is inversely proportional to the correlation length ${ }^{2} \xi_{G}$ of the fluctuating elastic constants ${ }^{3} G_{i}$. The complex frequencydependent sound velocities are given by

$$
\rho v_{T}(z)^{2}=G(z), \quad \rho v_{L}(z)^{2}=K_{\text {expt }}+\frac{4}{3} G(z) .
$$

Here $\rho=M N / V$ is the mass density, $M$ is the molecular mass, $N$ is the number of molecules, $V$ is the sample volume, and $K_{\text {expt }}$ is the experimentally determined bulk modulus. In our model the fluctuations of the local bulk moduli are considered to be negligible $[36,41]$.

It is easily checked from Eq. (3) that the relation $\chi_{L, T}(k, 0)=v_{L, T}(0)^{-2}$ holds. Therefore, in the static $\omega=0$ limit the descriptor $k_{e}$ becomes irrelevant. The zero-frequency limit of the frequency-dependent sound velocities are the experimentally measured sound velocities $v_{T}=v_{T}(0)=$ $\sqrt{G(0) / \rho}=\sqrt{G_{\text {expt }} / \rho}, \quad v_{L}=v_{L}(0)=\sqrt{\left[K_{\text {expt }}+\frac{4}{3} G_{\text {expt }}\right] / \rho}$. These quantities, together with $\rho$, are used as input for our fit procedure for obtaining the material descriptors. The dynamical input are the Raman spectra and the temperature dependent

\footnotetext{
${ }^{2}$ In a CPA version for correlated spatial fluctuations [48] the integral up to $k_{e}$ is replaced by an integral over the Green's functions times a $k$ dependent function $f(k)$, which is proportional to the the Fourier transform of the correlation function $C(k)$ of the fluctuating quantity. For correlation functions $C(r)$, which are exponentially decaying, $f(k)$ is equal to 1 for small $k$ values and then drops monotonically towards 0 near $k_{e}$.

${ }^{3}$ Comparing our obtained value of $k_{e}=0.345 \AA^{-1}$ for $\mathrm{SiO}_{2}$ with the correlation length $\xi_{G}=33 \AA$ obtained in $\mathrm{a} \mathrm{SiO}_{2}$ simulation [19] we have $k_{e} \xi_{G}=11.4$ as a proportionality factor.
}

specific heat. Within HET-CPA these vibrational spectra are obtained from the dynamic sound velocities $v_{L, T}(z)$, which are related to $G(z)$ via relation (4) [see the Appendix]. The latter is calculated self-consistently from the CPA equations (1), (3), and (4) with the condition $G(0)=G_{\text {expt }}$, using the log-normal distribution [2]. The output are the descriptors $G_{0}, \sigma^{2}$, and $k_{e}$. We emphasize that these descriptors have a well-defined physical meaning.

\section{B. Static descriptors and nonaffinity}

Before we discuss the material characterizations obtained by our fit procedure, we now establish a relation between the first two descriptors $G_{0}, \sigma^{2}$, and $G_{\text {expt. }}$. As shown in the Appendix, the CPA equation [Eq. (A10)] for the ratio $G(0) / G_{0}=G_{\text {expt }} / G_{0}$ does not depend further on $G_{0}$, so this ratio is a function of $\sigma^{2}$ with the parameter

$$
f(\kappa)=\frac{1}{3}\left(2+\frac{4}{4+3 \kappa}\right), \quad \kappa=\frac{K_{\text {expt }}}{G_{\text {expt }}},
$$

which varies very slowly with the ratio $\kappa$ between the limits $f(\infty)=2 / 3$ and $f(0)=1$. As further shown in the Appendix, in the small $\sigma^{2}$ limit the relation between $G_{\text {expt }} / G_{0}$ and $\sigma^{2}$ takes the form

$$
\frac{G_{\text {expt }}}{G_{0}}=1-\sigma^{2}\left(f(\kappa)-\frac{1}{2}\right) \equiv 1-n .
$$

It has been demonstrated [41,43] that the lowering of the macroscopic shear modulus with respect to the local average is due to nonaffine elasticity. Therefore we call $n$ (which increases almost linearly with $\sigma^{2}$ ) the nonaffine parameter. Then, relation (6), or its exact counterpart, Eq. (A10), combine the parameters $G_{0}$ and $\sigma^{2}$ into a single descriptor, i.e., $\sigma^{2}$ or $n$.

\section{RESULTS}

\section{A. Nonaffinity and ratio $K_{\text {expt }} / G_{\text {expt }}$}

In Fig. 1 we have plotted the ratio $G_{\text {expt }} / G_{0}=1-n$ vs $\sigma^{2}$ as obtained for a wide range of glassy materials. The full red line corresponds to the exact relation (A10) with $f(\kappa)=0.8$, which corresponds to $\kappa=2$, being a representative value for most of our materials. We further see from Fig. 1 that the CPA line for $f=0.8$ closely follows the straight dashed line given by Eq. (6) for $f=0.75$ in the regime $\sigma^{2}<1$, i.e., for low disorder strength.

\section{B. Spectral properties and the correlation length}

We now turn to the discussion of the influence of disorder on the spectral properties of glasses. We use the term "boson peak"4 relating to the prominent maximum of the reduced VDoS $g(\omega) / g_{D}(\omega)$ with $g_{D}(\omega)=3 \omega^{2} / \omega_{D}^{3}$. The Debye frequency is given by $\omega_{D}=k_{D} v_{D}$, where $k_{D}=\sqrt[3]{6 \pi^{2} N / V}$ is the Debye wave number and $v_{D}=v_{T}\left[\frac{1}{3}\left(2+\left[\kappa+\frac{4}{3}\right]^{-3 / 2}\right)\right]^{-1 / 3}$ is the Debye velocity. It has been demonstrated in the literature

\footnotetext{
${ }^{4}$ Very often in the literature the peak in the reduced Raman spectrum is also called boson peak, which causes some confusion.
} 
(a)

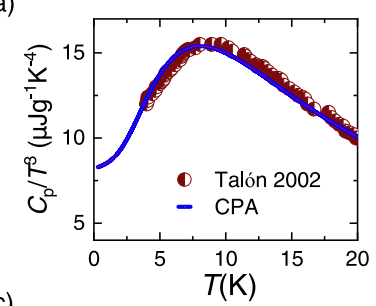

(c)

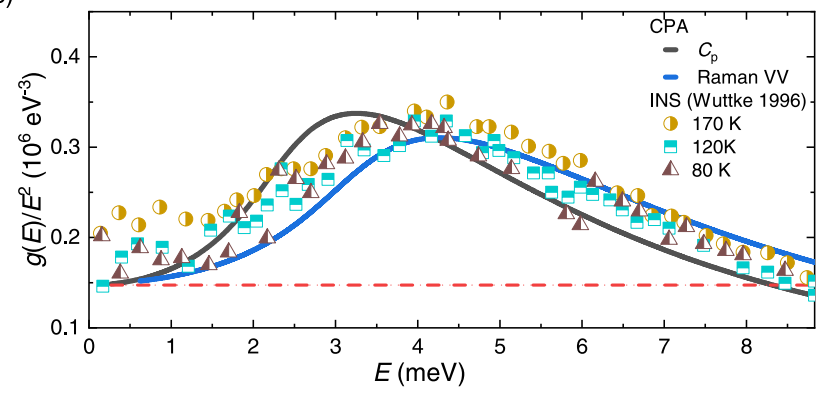

FIG. 2. Example of the CPA fit procedure for vitreous glycerol. The parametric descriptors $G_{0}, \sigma^{2}$, and $k_{e}$ are provided in the Supplemental Material Table S3 [49]. (a) The CPA fit is shown for the isobaric heat capacity $C_{p}$ with experimental data extracted from Ref. [50]. (b) The reduced Raman spectra $I_{V V, V H} / \omega[n(\omega)+1]$ extracted from HET-CPA for VV and VH geometries, respectively, with experimental data from Ref. [51] (175 K) and using the depolarization ratio of Kojima [52]. (c) The resulting reduced VDoS $g(\omega) / g_{D}(\omega)=g(\omega) \omega_{D}^{3} / 3 \omega^{2}$ are shown: Blue line: VDoS obtained from the $C_{p}$ data of (a), black line: VDoS obtained from the Raman spectra of (b). Symbols: VDoS data obtained by inelastic neutron scattering [53]. The dashed red line indicates the Debye level $3 / \omega_{D}^{3}$.

that the boson peak frequency $\omega_{b}$ coincides with the so-called Ioffe-Regel frequency at which the disorder-induced meanfree path becomes equal to the transverse-acoustic wavelength $[41,42]$. In the frequency range above $\omega_{b}$ the disorder dominates the vibrational spectrum

In Fig. 2 we demonstrate our fitting procedure for the archetypal example of glycerol. In Figs. 2(a) and 2(b) we plot the the reduced specific heat $C(T) / T^{3}$ and the reduced Raman intensity $I_{V V, V H}(\omega) / \omega[n(\omega)+1]$ (see the Appendix) together with the corresponding CPA fits. It is remarkable that the VDoSs obtained from the two data sets [displayed in Fig. 2(c)] almost agree with each other. Furthermore, there is a fair agreement with the VDoS data obtained by incoherent inelastic neutron scattering [53].

In Fig. 3 we have plotted the boson peak frequency $\omega_{b}$ vs the characteristic frequency $\omega_{0}=k_{e} v_{0}$, related to the descriptors $k_{e}$ and $G_{0}=\rho v_{0}^{2}$. We see that a precise linear relationship

$$
\omega_{b}=0.333 \omega_{0}
$$

is obtained. Because $\omega_{0} \propto \xi_{G}^{-1}$, we verify that the Ioffe-Regel crossover from wavelike vibrations to disorder dominated ones happens when the wavelength becomes of the order of the correlation length $\xi_{G}$. In other words, if the mesoscopic transverse sound velocity $v_{0}$ is used to convert from the length scale to the frequency scale, $\xi_{G}$ determines the boson peak frequency.

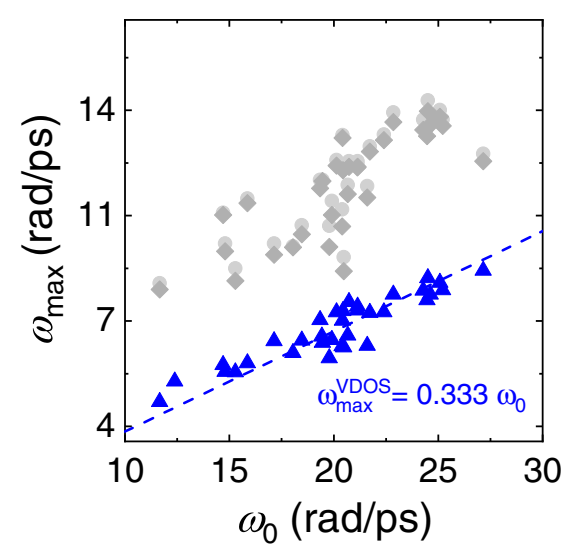

FIG. 3. Frequency positions of the maxima in the reduced Raman intensity (gray diamonds) and in the reduced VDoS (blue triangles) against $\omega_{0}=k_{e} v_{0}$.

In Fig. 4(a) we have plotted the height of the boson peak $\max \left\{g(\omega) / g_{D}(\omega)\right\}$ vs the relative boson peak position $\omega_{b} / \omega_{D}$. We obtain a power-law relationship

$$
\max \left\{g(\omega) / g_{D}(\omega)\right\}=0.46\left(\frac{\omega_{b}}{\omega_{D}}\right)^{-0.8}=1.11\left(\frac{\omega_{0}}{\omega_{D}}\right)^{-0.8}
$$

where the second equality is obtained from Eq. (7). An even more accurate power-law relationship is displayed in Fig. 4(b) for another dimensionless representation of the boson peak height:

$$
\omega_{0}^{3} \max \left\{g(\omega) / \omega^{2}\right\}=2.19\left(\frac{k_{e}}{k_{D}} \frac{1}{1-n}\right)^{2.42} .
$$

Relations (7), (8), and (9) relate unambiguously the position and the height of the boson peak to the descriptors $k_{e}$ and $G_{0}$.
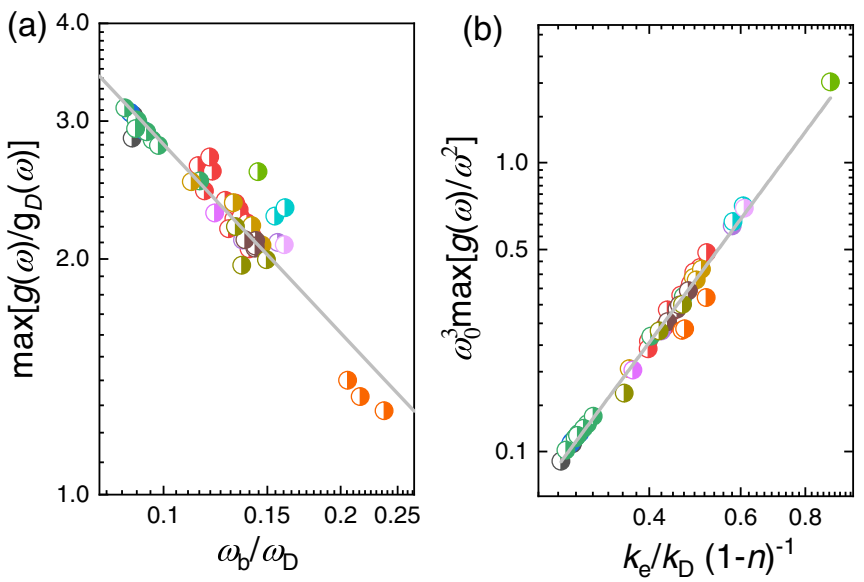

FIG. 4. (a) Double-logarithmic plot of the maximum of the reduced $\operatorname{VDoS} g(\omega) / g_{D}(\omega)$ vs $\omega_{b} / \omega_{D}$ with $g_{D}(\omega)=3 \omega^{2} / \omega_{D}^{3}$. (b) Double-logarithmic plot of the maximum of $\omega_{0}^{3} g(\omega) / \omega^{2}$ vs $\left(k_{e} / k_{D}\right)(1-n)^{-1}$. The gray lines indicate the exponent 0.8 (a) and $2.42(b)$, respectively. 

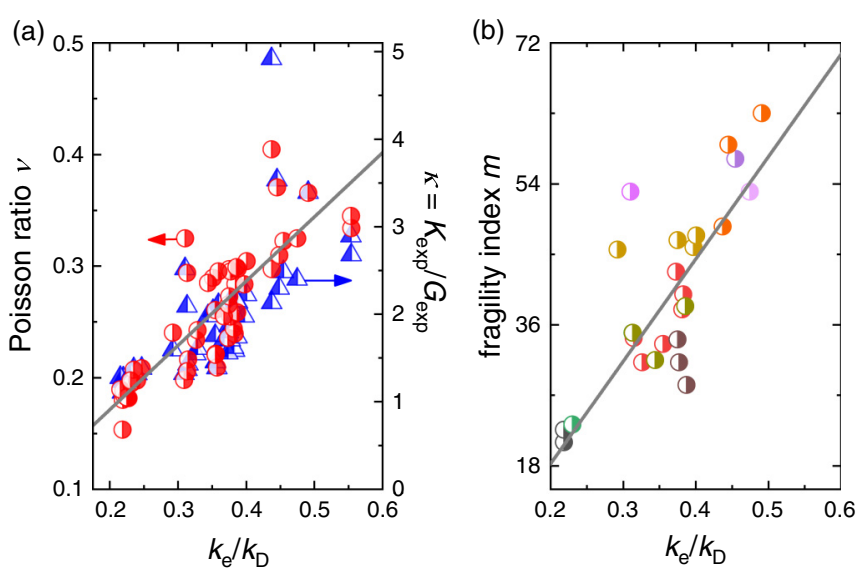

FIG. 5. (a) Poisson ratio $v$ (red circles, left scale) and $\kappa=$ $K_{\text {expt }} / G_{\text {expt }}$ (blue triangles, right scale) vs $k_{e} / k_{D}$. (b) fragility $m$ vs $k_{e} / k_{D}$. Symbol code as in Fig. 1 . The full lines are guides to the eye.

\section{Correlation length, Poisson ratio, and fragility}

In Fig. 5(a) we have plotted the Poisson ratio $v$ as well as $\kappa=K_{\text {expt }} / G_{\text {expt }}$ against $k_{e} / k_{D}$, showing that the empirical correlation can be better represented by a linear relation with $v$ than by a linear relation with $\kappa$. The correlation with $v$ is $v=0.06+0.56 k_{e} / k_{D}$.

The Poisson ratio is a rather important figure for designing new glass materials for applications [54,55]. For example, for $v>0.3$ one expects a brittle-to-ductile transition, therefore, values of $k_{e} / k_{D}>0.5$ would point to a ductile material. This makes sense intuitively, as a large value of $\xi_{G} k_{D}$ will lead to a rather rigid network.

A further characteristic property of glasses is the fragility index $m$ of its corresponding liquid [7], which is the relative slope of the logarithm of the temperature dependence of the viscosity $\eta$ near the glass transition temperature $T_{g}$. It is defined by $m=\partial \log _{10} \eta /\left.\partial\left[T_{g} / T\right]\right|_{T=T_{g}}$. Novikov and Sokolov $[56,57]$ proposed an empirical relationship between the Poisson ratio and $m$ for a number of nonconducting glasses. This had been challenged by Yannopoulos and Johary [58], presenting a large number of exceptions. Later it became clear that such relationships appear only in certain material classes [59]. Very recently Østergaard et al. collected viscosity and elasticity data for a very large number of glasses [55]. They have shown that rather than a correlation a trend exists for larger $m$ values in materials with larger $v$ values, see Fig. 6. In Fig. 5(b) we have plotted $m$ against $k_{e} / k_{D}$ of those materials involved in our present study for which viscosity data is available in the literature. Indeed, we find a weak but notable positive correlation between $m$ and the ratio of $k_{e} / k_{D}$. If we combine this correlation with the one shown in Fig. 5(a) we find $m=218 v-17$, which is inserted as a red dotted line into the trend picture of Østergaard et al. in Fig. 6. We add Sokolov's correlation for nonmetals [59] $m=29 \kappa-12$. It is seen that it also fits into Østergaard's trend. For our classification it is important to note that large $k_{e} / k_{D}$ values prevail for fragile materials.

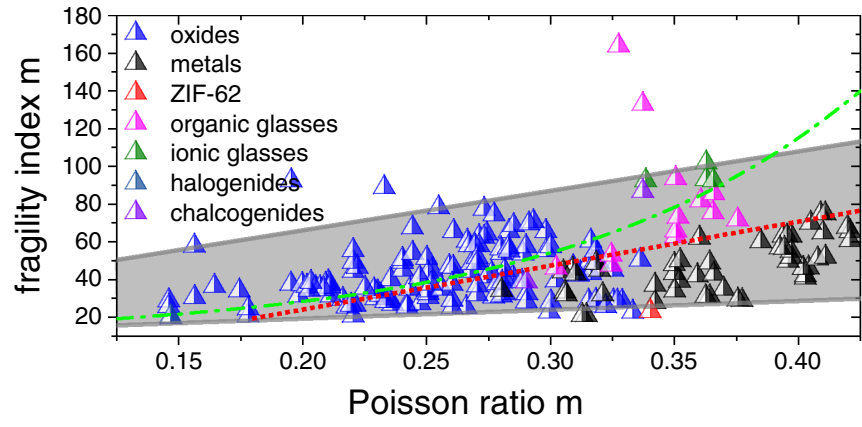

FIG. 6. Symbols: fragility $m$ vs Poisson ratio $v$ as compiled by Østergaard et al. [55]. Dotted red line: Present empirical correlation as obtained from the data of Fig. 5. Dash-dotted green line: Empirical correlation obtained by Sokolov [56,57,59] for nonmetallic glasses. The gray-shaded region marks the overall trend of reported experimental data.

\section{DISCUSSION}

We have already emphasized that our disorder classification essentially reduces to two descriptors, the disorder parameter $\sigma^{2}$, which is proportional to the nonaffinity $n=$ $1-G_{\text {expt }} / G_{0}$ and the upper momentum cutoff $k_{e}$, which is inversely proportional to the correlation length $\xi_{G}$ of the elasticity fluctuations.

The nonaffine reduction of the macroscopic shear modulus $G_{\text {expt }}$, which appears in all types of glass, may be interpreted in terms of acoustic percolation [47]. The local sound propagation is fastest in regions of high rigidity and, in turn, the strain energy is localized in the soft, nonaffine regions. Under shear, the rigid backbone regions then enclose the nonaffine soft regimes, which feature rotational patterns. Metallic glasses with low $\sigma^{2}$ exhibit less pronounced nonaffine features. In the more interconnected morphologies, softer modes become a serious obstacle for sound propagation, leading to an enhanced acoustic path length and therefore a reduction of the effective velocity. This is in perfect analogy of the percolational reduction of the conductivity in disordered networks [60], and is the reason for the remarkable relation between nonaffinity $n$ and $\sigma^{2}$.

The remaining descriptor, the correlation length $\xi_{G}$ of the spatial rigidity fluctuations, features strong correlations with the frequency position $\omega_{b}$ and the height of the boson peak. On the other hand, it is related to the ratio of the shear and bulk modulus $\kappa$, hence, to the Poisson ratio $v$ and-trendwise-to the fragility index $m$.

Let us start discussing the relation $\omega_{b}=0.333 \omega_{0}$, revealed in Fig. 3. As $\omega_{0}=k_{e} \sqrt{G_{0} / \rho}$ is inversely proportional to $\xi_{G}$, so is the boson peak position. Such a correlation between $\omega_{b}$ and the inverse of a structure-related correlation function was already conjectured in the early days of the discussion of the boson peak $[61,62]$, but had never been unambiguously quantified. The velocity connecting the boson peak frequency and the inverse correlation length turns out to be not related to the macroscopic transverse sound velocity $v_{T}$ but to the average local one, $v_{0}$, i.e., the one related to the typical local shear modulus. Only when a proportionality with $v_{T}$ is enforced, a 
structure-related prefactor appears:

$$
\omega_{b} \propto \omega_{0} \propto \frac{1}{\xi_{G}} \frac{v_{T}}{\sqrt{1-n}} .
$$

We see, if $v_{T} / \xi_{G}$ is used instead of $v_{0} / \xi_{G}$, a correction factor $[1-n]^{-1 / 2}$ appears, ${ }^{5}$ which is similar to the empirical "shape factor," introduced by Duval et al. [62] in order to account for specific material properties so as to improve the correlation between $\omega_{b}$ and $v_{T} / \xi_{G}$. Instead of some assumed shape of rigid inclusions [62], our correction factor accounts for the nonaffinity and, by the CPA equivalence (6), to the disorder strength.

Summarizing the trends displayed in Fig. 6, and combining the findings of Figs. 5 and 1, we may say that with increasing liquid fragility, the correlation length (relative to the intermolecular distance) decreases and nonaffinity (weakly) increases. The former observation is a strong confirmation for the correlation between liquid heterogeneity and heterogeneity in the solid (glassy) state which was previously found for molecular glass formers and colloidal suspensions [63,64], where the growing size of correlated regions was identified as the origin of the dynamic slow down during glass formation (further support may be found in the correlation between liquid fragility and structural fragility which was discovered for metallic glasses [65]). It is, indeed, known that structures presenting an abundance of soft elastic modes tend to have a greater length scale of shear fluctuation $\xi_{G}$ and are strong (low Poisson ratio). Previous modeling studies [66] found that the amplitude of the boson peak (in Debye units) correlates well with the inverse of the fragility $1 / \mathrm{m}$. Our data provide experimental confirmation for this observation, as relation (8) implies $\max \left\{g(\omega) / g_{D}(\omega)\right\} \propto\left(k_{e} / k_{D}\right)^{-0.8} \propto m^{-0.8}$.

The metal-organic framework glass represents a particular case. A glass formed from ZIF-62, termed $a_{g}$-ZIF-62, was obtained through structural collapse of a nanoporous crystal at temperatures only mildly above $T_{m}$. As a relic of the crystalline state, the resulting glass contains pores on the scale of $0.5 \mathrm{~nm}$ [67], representing a disperse morphology of soft modes. In terms of the governing length scales and the function of $f(\kappa)$, their properties are similar to metallic glasses. However, among all the considered glass chemistries, they exhibit the highest nonaffinity and the highest disorder parameter.

\section{CONCLUSION}

In conclusion, we implemented heterogeneous-elasticity theory in conjunction with the coherent-potential approximation using experimental input from a broad variety of glass chemistries in order to extract the vibrational density of states and the probability distribution density of the local shear modulus of glassy materials. The exclusive use of physically

\footnotetext{
${ }^{5}$ If $\xi_{G}$ and $v_{T}$ would not be influenced by the disorder parameter $\sigma^{2}$, and because $n$ is directly proportional to $\sigma^{2}$, Eq. (10) would imply that $\omega_{b}$ would shift to higher frequencies with increasing $\sigma^{2}$. This is in contradiction to the theoretical prediction of HET [47] and counterintuitive. We checked from our data that indeed $\omega_{b}$ does not increase with $\sigma^{2}$.
}

meaningful fitting parameters enables widespread application of our approach to real-world materials, revealing remarkable trends which appear to hold across all classes of glass (despite pronounced differences in bond directionality and localization).

In analyzing our data, we extracted three universal descriptors of disorder and the statistics of elastic heterogeneity: The typical shear modulus $G_{0}$ and the disorder parameter $\sigma^{2}$, which characterize the log-normal distribution function of local elasticity, and the momentum cutoff $k_{e}$, which is inversely proportional to the correlation length scale $\xi_{G}$. Using experimental information on the macroscopic shear modulus $G_{\text {expt }}$, we find a one-to-one relation between the nonaffinity $n=1-G_{\text {expt }} / G_{0}$ and $\sigma^{2}$. This leaves only two independent descriptors, the disorder parameter $\sigma^{2}$ and the cutoff wave number $k_{e}$, viz. the correlation length. We found a further, linear correlation between the frequency $\omega_{0} \propto k_{e} \sqrt{G_{0}}$ and the boson peak position $\omega_{b}$, and a power-law relation between the boson peak amplitude and frequency positions in Debye units. Furthermore, we revealed a correlation between $k_{e}$ and the Poisson ratio and, trendwise, liquid fragility.

It remains to be explored how disordered materials derived from techniques other than liquid quenching (e.g., the prolific superstrong glasses [68] or pressure-amorphized crystals [67]) would fall into the observed trends. Furthermore, extending the range of observation to materials at the extremes of disorder and with enhanced nonaffinity, respectively, would be a significant step in understanding the further role of structural heterogeneity on macroscopic properties through refining the discovered relations, for example, by considering pressurized or autoclaved glasses and mesoporous hybrids beyond the very few examples which are known today. The link between HET, disorder classification by use of vibrational spectra, and direct structural data obtained outside of computer models remains elusive at present. Nascent experimental techniques (for example, in electron microscopy) might provide routes for direct imaging of elastic heterogeneity for a broader range of materials so as to establish a comprehensive understanding of analytical descriptors and the underlying statistics of disorder.

Such insight will open new routes for specific and independent tailoring of nonaffinity and structural heterogeneity towards macroscopic properties. Aside mechanical and vibrational behavior, this also concerns the transport coefficients of sound propagation, heat, and ion conduction.

\section{ACKNOWLEDGMENTS}

This project has received funding from the European Research Council (ERC) under the European Union's Horizon 2020 research and innovation program (L.W., ERC Grant UTOPES, Grant agreement No. 681652). T.D.B acknowledges the Royal Society for a University Research Fellowship (UF150021) and the Leverhulme Trust for a Philip Leverhulme Prize. L.W. conceived of the experiments and wrote the manuscript together with W.S., W.S., and Z.P. L.W. developed the extended HET-CPA. Z.P. was responsible for numerical implementation and data fits. O.B. was responsible for the Raman spectroscopic analysis. O.B., S.S., A.K., R.L., T.D.B., and G.W. provided sample material and acquired the supporting physical data, including low-temperature heat 
capacity measurements. All authors participated in data analysis and evaluation, and were involved in the discussion and manuscript revisions. The authors declare no competing interests.

\section{APPENDIX: MATERIALS AND METHODS}

\section{Materials}

The glass samples used in this study were chosen on the condition of individual material homogeneity and availability of general physical data so as to cover a broad range of materials with variable bonding character and variations in fundamental structural motifs and network dimensionality. Details on the studied compositions are provided in the Supplementary Material (see, also, Refs. [69-105] therein), Table S1 [49], together with their glass transition temperature $T_{g}$, liquid fragility index $m$, mass density $\rho$, and calculated values of the volume density of bond energy $\left\langle U_{0} / V_{0}\right\rangle$ and of the atomic packing density $C_{g}$. The experimental (macroscopic) shear, bulk and Young's modulus $G_{\text {expt }}, K_{\text {expt }}$, and $E_{\text {expt }}$, respectively, and Poisson's ratio $v$ are given in Table S2 [49]. Most glasses were prepared by conventional melt-quenching techniques. Some commercial samples were used as obtained, including a standard soda-lime silicate glass (Marienfeld Superior, Paul Marienfeld, Lauda-Königshofen, Germany), a low-alkali borosilicate glass (Borofloat-33, Schott TGS, Jena, Germany), two variants of vitreous silica [Suprasil, Heraeus Quarzglas, Hanau, Germany, and R300, prepared by a modified chemical vapor deposition route (MCVD), respectively], and a range of optical glasses (Schott, Mainz, Germany). Detailed information on the fabrication procedures are provided in the references given in Tables S1 and S2 [49]. All materials were used in the form of disks with a thickness of 1-5 mm and a diameter of $2 \mathrm{~mm}$, polished to optical grade using $\mathrm{CeO}_{2}$ suspensions. X-ray diffraction (MiniFlex600, Rigaku) was conducted on all samples in order to confirm the absence of any detectable crystalline species. The glass transition temperatures were determined by differential scanning calorimetry (Netzsch STA 449 F3 Jupiter) in flowing nitrogen, employing heating rates of $20 \mathrm{~K} / \mathrm{min}$ for the MeP, SP, and $\mathrm{a}_{g}$-ZIF62 glasses, $40 \mathrm{~K} / \mathrm{min}$ for $\mathrm{Cu}_{46} \mathrm{Zr}_{46} \mathrm{Al}_{8}(40 \mathrm{~K} / \mathrm{min})$, and 10 $\mathrm{K} / \mathrm{min}$ for all other glasses. Density data were obtained with an Archimedes balance using distilled water or dry ethanol as immersion media. Where not already available from previous studies (for references see Table S2 [49]), the macroscopic (effective) elastic properties were analyzed through ultrasonic echography at room temperature. The longitudinal and transversal sound velocities $v_{L}$ and $v_{T}$ were derived from the corresponding sound wave propagation times (recorded with an accuracy of \pm 1 ns by piezoelectric transducers operating at frequencies of 8 to $12 \mathrm{MHz}$ ) and the exact thickness of the polished glass plates (determined with an accuracy of $\pm 2 \mu \mathrm{m}$ using a micrometer screw). The macroscopic elastic moduli were calculated from these values.

\section{Vibrational spectra}

Raman scattering spectroscopy was carried-out using a Renishaw inVia confocal Raman microscope equipped with a low-frequency notch filter covering the frequency range of $0-200 \mathrm{~cm}^{-1}$. Samples were excited with an argon ion laser operating at $514.5 \mathrm{~nm}$. The signal was collected with a CCD camera at a spectral resolution of $2 \mathrm{~cm}^{-1}$, using a grating with 2400 lines $/ \mathrm{mm}$ and a $50 \times$ microscope objective. All spectra were collected in two polarization geometries, $\mathrm{VV}$ and $\mathrm{VH}$, by means of a polarizer/half-wave plate setup inserted in the laser beamline between the notch filter and the monochromator. For the chalcogenides and the metallic glasses, the vibrational properties were investigated by heat capacity $\left(C_{p}\right)$ measurements at low temperature. For the chalcogenides this was a precautious measure because the relatively harsh Raman excitation conditions might have induced experimental artifacts, which would compromise subsequent data analyses. For the metallic glasses, they are generally unsuitable for Raman spectroscopy because first-order Raman bands result from a change in the polarizability of the molecule and metallic materials are infinitely polarizable. Further low-temperature heat capacity data on vitreous silica and on the aluminosilicate glasses (AS) were extracted from a previous study [106]. $C_{p}$ analyses of the chalcogenide glasses $\mathrm{As}_{38} \mathrm{Se}_{62}$ and $\mathrm{GeSe}_{4}$, and of the metallic glasses $\mathrm{Pd}_{40} \mathrm{Ni}_{40} \mathrm{P}_{20}$ and $\mathrm{Zr}_{52.5} \mathrm{Cu}_{17.9} \mathrm{Ni}_{14.6} \mathrm{Al}_{10} \mathrm{Ti}_{5}$ were conducted on a physical property measurement system (PPMS, Quantum Design) at temperatures down to approximately $2.3 \mathrm{~K}$. $C_{p}$ data on vitreous $\mathrm{As}_{2} \mathrm{~S}_{3}$ [107] and $\mathrm{Cu}_{46} \mathrm{Zr}_{46} \mathrm{Al}_{8}$ [108] were taken from literature, as were all experimental data of glycerol [50-53].

\section{CPA calculation of spectral functions}

From the CPA equations (1), (3), and (4) the dynamic sound velocities $v_{L, T}(z)$ are obtained via $G(z)$. From these quantities the VDoS is obtained as

$$
g(\omega)=\frac{2 \omega}{3 \pi} \frac{3}{k_{D}^{3}} \int_{0}^{k_{D}} d k k^{2}\left[\frac{1}{-z^{2}+k^{2} v_{L}^{2}(z)}+2 \frac{1}{-z^{2}+k^{2} v_{T}^{2}(z)}\right] .
$$

Here $k_{D}=\sqrt[3]{6 \pi^{2} N / V}$ is the Debye wave number. The temperature dependent specific heat is given by

$$
C_{p}(T)=A_{c} \int_{0}^{\infty} g(\omega) \frac{\omega^{2}}{T^{2}} \frac{e^{\frac{\hbar \omega}{k_{B} T}}}{\left(e^{\frac{\hbar \omega}{k_{B} T}}-1\right)^{2}} d \omega,
$$

$A_{c}$ is a prefactor and $\hbar$ and $k_{B}$ are Planck's and Boltzmann's constants. The depolarized $(V H)$ and polarized $(V V)$ Raman spectra are $[31,36,46]$

$$
I_{V H}(\omega)=A_{V H}[n(\omega)+1]\left(\frac{4}{3}\left[\chi_{L}\left(k_{a}, \omega\right)\right]^{\prime \prime}+2\left[\chi_{T}\left(k_{a}, \omega\right)\right]^{\prime \prime}\right),
$$

$$
I_{V V}(\omega)=A_{V V}[n(\omega)+1]\left[\chi_{L}\left(k_{a}, \omega\right)\right]^{\prime \prime}+\frac{4}{3} I_{V H}(\omega) .
$$

$A_{V H}$ and $A_{V V}$ are prefactors and $k_{a}$ is a cutoff related to the correlation length of the Pockels-constant fluctuations. $n(\omega)=\left[e^{\hbar \omega / k_{B} T}-1\right]^{-1}$ is the boson occupation number (to which the boson peak owes its name). In addition to $\sigma^{2}, G_{0}, k_{e}$ the Raman spectra feature two further descriptors ("Raman descriptors"), which are the wave number cutoff $k_{a} \propto \xi_{a}^{-1}$, where $\xi_{a}$ is the correlation length of the Pockels-constant fluctuations and the amplitude ratio $A_{V V} / A_{V H}$ of the two types 
of Pockels-constant correlation functions [31]. As in previous studies $[31,33,46], k_{a}$ turned out to be very near to $k_{e}$, see Table S3 [49].

\section{CPA equation for $\omega=0$}

The CPA equation for $\omega=0$ is

$$
\begin{aligned}
0 & =\left\langle\frac{G_{i}-G(0)}{1+\frac{1}{3}\left[G_{i}-G(0)\right] \Lambda(0)}\right\rangle_{P} \\
& \Leftrightarrow\left\langle\frac{G_{i}}{1+\frac{1}{3}\left[G_{i}-G(0)\right] \Lambda(0)}\right\rangle_{P} \\
& =G(0)\left\langle\frac{1}{1+\frac{1}{3}\left[G_{i}-G(0)\right] \Lambda(0)}\right\rangle_{P} \\
& =G(0)\left\langle\frac{1+\frac{1}{3}\left[G_{i}-G(0)\right] \Lambda(0)-\frac{1}{3}\left[G_{i}-G(0)\right] \Lambda(0)}{1+\frac{1}{3}\left[G_{i}-G(0)\right] \Lambda(0)}\right\rangle_{P} \\
& =G(0) \equiv G_{\text {expt }}
\end{aligned}
$$

We have

$$
\frac{1}{3} \Lambda(0)=\frac{1}{3}\left(\frac{2}{G_{\text {expt }}}+\frac{4}{3} \frac{1}{K_{\text {expt }}+\frac{4}{3} G_{\text {expt }}}\right)=f(\kappa) \frac{1}{G_{\text {expt }}},
$$

with

$$
f(\kappa)=\frac{1}{3}\left(2+\frac{4}{4+3 \kappa}\right) \quad \kappa=\frac{K_{\mathrm{expt}}}{G_{\mathrm{expt}}} .
$$

We note that $f(\kappa)$ is a very slowly varying function with

$$
f(\infty)=\frac{2}{3} \leqslant f(\kappa) \leqslant f(0)=1 .
$$

We now introduce dimensionless variables into the second version of Eq. (A6): $g_{i}=G_{i} / G_{0}$ and $Q=G_{\text {expt }} / G_{0}$ and obtain

$$
\begin{aligned}
Q= & \frac{1}{G_{0} \sqrt{2 \pi} \sigma} \int_{0}^{\infty} d G_{i} \frac{1}{G_{i}} e^{-\frac{1}{2 \sigma^{2}}\left[\ln \left(G_{i} / G_{0}\right)\right]^{2}} \\
& \times \frac{G_{i}}{1+f(\kappa)\left[\frac{g_{i}}{Q}-1\right]} \\
= & \frac{1}{\sqrt{2 \pi} \sigma} \int_{0}^{\infty} d g_{i} \frac{e^{-\frac{1}{2 \sigma^{2}}\left[\ln \left(g_{i}\right)\right]^{2}}}{1+f(\kappa)\left[\frac{g_{i}}{Q}-1\right]} .
\end{aligned}
$$

We see that $Q$ is an implicit function of $\sigma^{2}$ (and vice versa) with $f(\kappa)$ as parameter.

\section{CPA calculation of $G_{\text {expt }}$ in the small-disorder limit}

We now define new deviatoric moduli:

$$
G_{\text {expt }}=\left\langle G_{i}\right\rangle_{P}-\Sigma, \quad G_{i}=\left\langle G_{i}\right\rangle_{P}-\Delta_{i} .
$$

The CPA equation (A6) becomes

$$
0=\left\langle\frac{\Delta_{i}-\Sigma}{1-\frac{f(\kappa)}{G_{\text {expt }}}\left[\Delta_{i}-\Sigma\right]}\right\rangle_{P} \Leftrightarrow \Sigma=\left\langle\frac{\Delta_{i}}{1-\frac{f(\kappa)}{G_{\text {expt }}}\left[\Delta_{i}-\Sigma\right]}\right\rangle_{P} .
$$

We now treat $\Delta_{i}$ and $\Sigma$ to be of order $\sigma$ and obtain to lowest (quadratic) order

$$
\Sigma \approx \frac{f(\kappa)}{\left\langle G_{i}\right\rangle_{P}}\left\langle\Delta_{i}^{2}\right\rangle_{P}
$$

Therefore,

$$
\frac{G_{\text {expt }}}{\left\langle G_{i}\right\rangle_{P}}=1-\frac{\Sigma}{\left\langle G_{i}\right\rangle_{P}} \approx 1-f(\kappa) \frac{\left\langle\Delta_{i}^{2}\right\rangle_{P}}{\left\langle G_{i}\right\rangle_{P}^{2}}=1-f(\kappa)\left(e^{\sigma^{2}}-1\right)
$$

and finally

$$
\begin{aligned}
Q & =\frac{G_{\mathrm{expt}}}{G_{0}}=\frac{G(0)}{\left\langle G_{i}\right\rangle_{\mathrm{geo}}}=e^{\frac{1}{2} \sigma^{2}}\left[1-f(\kappa)\left(e^{\sigma^{2}}-1\right)\right] \\
& \approx 1-\left(f(\kappa)-\frac{1}{2}\right) \sigma^{2} .
\end{aligned}
$$

\section{Numerical implementation}

The CPA equation can be solved numerically within a few iterations. The resulting solution, the frequencydependent shear modulus $G(z)$, was then used to obtain the vibrational density of states $g(\omega)$. Fits were constructed in terms of a least-square minimization of the difference between the experimental data and the corresponding theoretical predictions of Eqs. (A2)-(A4). There were, therefore, two independent fitting procedures involved in this work: the $C_{p}$ fit and the Raman fit. The $C_{p}$ fit produced $\left(\sigma, G_{0}, k_{e}\right)$ while taking $\left[v_{L}, v_{T}, k_{D}, C_{p}(2.3<T<20 \mathrm{~K})\right]$ for input. The Raman fit produced $\left(\sigma, G_{0}, k_{e}, k_{a}, A_{21}\right)$ by using $\left[v_{L}, v_{T}, k_{D}, I_{V V}(\omega), I_{V H}(\omega)\right]$ for input, where $A_{21}=$ $A_{V H} / A_{V V}$. The frequency ranges for Raman spectra were selected from $15 \mathrm{~cm}^{-1}$ below the Raman boson peak up to 100 $\mathrm{cm}^{-1}$. Since the fitting problems are ill-posed, we employed Tikhonov regularization. As the criterion for identifying the optimized regularization parameter for the $C_{p}$ fit, we used the minimum of the smoothness parameter $M$ of the reduced VDoS ( $M$ increases sharply if the reduced VDoS starts to oscillate). In this way, an independent, individually optimized regularization parameter was attributed to each glass data set. For the Raman fit, the goodness of fit $(G O F)$ was additionally used. $G O F$ evaluates the maximum curve length of an experimental Raman spectrum which fits to the predicted one. It is noteworthy that, although we restricted the Raman fit input to the spectral range of $<100 \mathrm{~cm}^{-1}$, the obtained fits of most samples showed good agreement to experimental data also up to higher frequency $\left(<200 \mathrm{~cm}^{-1}\right)$. The full evaluation procedure was scripted in order to produce independent and automated fits for Raman and $C_{p}$ experimental data, respectively. In this way, the VDoS was derived independently from two different experimental data sets. 
[1] W. H. Zachariasen, J. Am. Chem. Soc. 54, 3841 (1932).

[2] D. Turnbull and M. H. Cohen, J. Chem. Phys. 34, 120 (1961).

[3] Y. Cheng and E. Ma, Prog. Mater. Sci. 56, 379 (2011).

[4] G. Biroli and J. P. Garrahan, J. Chem. Phys. 138, 12A301 (2013).

[5] L. Berthier and G. Biroli, Rev. Mod. Phys. 83, 587 (2011).

[6] M. D. Ediger, Annu. Rev. Phys. Chem. 51, 99 (2000).

[7] C. A. Angell, Science 267, 1924 (1995).

[8] B. Ruta, G. Monaco, V. Giordano, F. Scarponi, D. Fioretto, G. Ruocco, K. Andrikopoulos, and S. Yannopoulos, J. Phys. Chem. B 115, 14052 (2011).

[9] A. Makishima and J. D. Mackenzie, J. Non-Cryst. Solids 17, 147 (1975).

[10] Y. Shi, A. Tandia, B. Deng, S. R. Elliott, and M. Bauchy, Acta Mater. 195, 252 (2020).

[11] E. D. Cubuk, R. Ivancic, S. S. Schoenholz, D. Strickland, A. Basu, Z. Davidson, J. Fontaine, J. L. Hor, Y.-R. Huang, Y. Jiang et al., Science 358, 1033 (2017).

[12] A. Nicolas, E. E. Ferrero, K. Martens, and J.-L. Barrat, Rev. Mod. Phys. 90, 045006 (2018).

[13] B. Wang, Y. Yu, M. Wang, J. C. Mauro, and M. Bauchy, Phys. Rev. B 93, 064202 (2016).

[14] O. Benzine, S. Bruns, Z. Pan, K. Durst, and L. Wondraczek, Adv. Sci. 5, 1800916 (2018).

[15] E. J. Frankberg, J. Kalikka, F. G. Ferré, L. Joly-Pottuz, T. Salminen, J. Hintikka, M. Hokka, S. Koneti, T. Douillard, B. Le Saint et al., Science 366, 864 (2019).

[16] J. P. Wittmer, A. Tanguy, J.-L. Barrat, and L. Lewis, Europhys. Lett. 57, 423 (2002).

[17] B. A. DiDonna and T. C. Lubensky, Phys. Rev. E 72, 066619 (2005).

[18] F. Leonforte, R. Boissière, A. Tanguy, J. P. Wittmer, and J.-L. Barrat, Phys. Rev. B 72, 224206 (2005).

[19] F. Leonforte, A. Tanguy, J. P. Wittmer, and J.-L. Barrat, Phys. Rev. Lett. 97, 055501 (2006).

[20] M. Tsamados, A. Tanguy, C. Goldenberg, and J.-L. Barrat, Phys. Rev. E 80, 026112 (2009).

[21] J. Ding, Y.-Q. Cheng, H. Sheng, M. Asta, R. O. Ritchie, and E. Ma, Nat. Commun. 7, 13733 (2016).

[22] W. A. Phillips and A. Anderson, Amorphous Solids: LowTemperature Properties (Springer, Berlin, 1981), Vol. 24.

[23] U. Buchenau, N. Nücker, and A. J. Dianoux, Phys. Rev. Lett. 53, 2316 (1984).

[24] B. Frick and D. Richter, Science 267, 1939 (1995).

[25] A. I. Chumakov, I. Sergueev, U. van Bürck, W. Schirmacher, T. Asthalter, R. Rüffer, O. Leupold, and W. Petry, Phys. Rev. Lett. 92, 245508 (2004).

[26] J. Wuttke, W. Petry, G. Coddens, and F. Fujara, Phys. Rev. E 52, 4026 (1995).

[27] G. Monaco and V. M. Giordano, Proc. Natl. Acad. Sci. 106, 3659 (2009).

[28] G. Baldi, A. Fontana, G. Monaco, L. Orsingher, S. Rols, F. Rossi, and B. Ruta, Phys. Rev. Lett. 102, 195502 (2009).

[29] G. Baldi, V. M. Giordano, G. Monaco, and B. Ruta, Phys. Rev. Lett. 104, 195501 (2010).

[30] G. Baldi, V. M. Giordano, and G. Monaco, Phys. Rev. B 83, 174203 (2011).

[31] B. Schmid and W. Schirmacher, Phys. Rev. Lett. 100, 137402 (2008), see references therein for an extended list of Ramanspectroscopy papers.
[32] S. Caponi, S. Corezzi, D. Fioretto, A. Fontana, G. Monaco, and F. Rossi, Phys. Rev. Lett. 102, 027402 (2009).

[33] A. Schulte, W. Schirmacher, B. Schmid, and T. Unruh, J. Phys. Condens. Matter 23, 254212 (2011).

[34] M. A. Ramos, C. Talón, and S.Vieira, J. Non-Cryst. Solids 307-310, 80 (2002).

[35] M. A. Ramos, Low Temperature Phys. 46, 104 (2020).

[36] W. Schirmacher, T. Scopigno, and G. Ruocco, J. Non-Cryst. Solids 407, 133 (2015).

[37] T. Nakayama, Rep. Prog. Phys. 65, 1195 (2002).

[38] M. I. Klinger, Phys. Rep. 292, 111 (2010).

[39] A. I. Chumakov, G. Monaco, A. Monaco, W. A. Crichton, A. Bosak, R. Ruffer, A. Meyer, F. Kargl, L. Comez, D. Fioretto, H. Giefers, S. Roitsch, G. Wortmann, M. H. Manghnani, A. Hushur, Q. Williams, J. Balogh, K. Parlinski, P. Jochym, and P. Piekarz, Phys. Rev. Lett. 106, 225501 (2011).

[40] A. I. Chumakov, G. Monaco, A. Fontana, A. Bosak, R. P. Hermann, D. Bessas, B. Wehinger, W. A. Crichton, M. Krisch, R. Ruffer, G. Baldi, G. Carini, G. Carini, G. DAngelo, E. Gilioli, G. Tripodo, M. Zanatta, B. Winkler, V. Milman, K. Refson, M. T. Dove, N. Dubrovinskaia, L. Dubrovinsky, R. Keding, and Y. Z. Yue, Phys. Rev. Lett. 112, 025502 (2014).

[41] A. Marruzzo, W. Schirmacher, A. Fratalocchi, and G. Ruocco, Sci. Rep. 3, 1407 (2013).

[42] H. Shintani and H. Tanaka, Nat. Mater. 7, 870 (2008).

[43] H. Mizuno, S. Mossa, and J.-L. Barrat, Phys. Rev. E 87, 042306 (2013).

[44] W. Schirmacher, Europhys. Lett. 73, 892 (2006).

[45] W. Schirmacher, G. Ruocco, and T. Scopigno, Phys. Rev. Lett. 98, 025501 (2007).

[46] B. Schmid, Diploma thesis, Technische Universität München, 2007.

[47] S. Köhler, G. Ruocco, and W. Schirmacher, Phys. Rev. B 88, 064203 (2013).

[48] R. Zimmermann and C. Schindler, Phys. Rev. B 80, 144202 (2009).

[49] See Supplemental Material at http://link.aps.org/supplemental/ 10.1103/PhysRevB.104.134106 for detailed data on glass compositions, physical properties and fit results.

[50] C. Talón, Q. W. Zou, M. A. Ramos, R. Villar, and S. Vieira, Phys. Rev. B 65, 012203 (2001).

[51] T. Uchino and T. Yoko, Science 273, 480 (1996).

[52] S. Kojima, Phys. Rev. B 47, 2924(R) (1993).

[53] J. Wuttke, W. Petry, and S. Pouget, J. Chem. Phys. 105, 5177 (1996).

[54] G. N. Greaves, A. L. Greer, R. S. Lakes, and T. Rouxel, Nat. Mater. 10, 823 (2011).

[55] M. B. Østergaard, S. R. Hansen, K. Januchta, T. To, S. J. Rzoska, M. Bockowski, M. Bauchy, and M. M. Smedskjaer, Materials 12, 2439 (2019).

[56] M. N. Novikov and A. P. Sokolov, Nature (London) 431, 961 (2004).

[57] V. N. Novikov, Y. Ding, and A. P. Sokolov, Phys. Rev. E 71, 061501 (2005).

[58] S. N. Yannopoulos and G. P. Johari, Nature (London) 442, E7 (2006).

[59] V. N. Novikov and A. P. Sokolov, Phys. Rev. B 74, 064203 (2006).

[60] A. I. Efros and B. I. Shklovsk, Electronic Properties of Doped Semiconductors (Springer, Heidelberg, 1984). 
[61] S. Elliott, Europhys. Lett. 19, 201 (1992).

[62] E. Duval, A. Boukenter, and T. Achibat, J. Phys. Condens. Matter 2, 10227 (1990).

[63] X. Qiu and M. Ediger, J. Phys. Chem. B 107, 459 (2003).

[64] L. Berthier, G. Biroli, J.-P. Bouchaud, L. Cipelletti, D. El Masri, D. L'Hôte, F. Ladieu, and M. Pierno, Science 310, 1797 (2005).

[65] N. A. Mauro, M. Blodgett, M. L. Johnson, A. J. Vogt, and K. F. Kelton, Nat. Commun. 5, 4616 (2014).

[66] L. Yan, G. Düring, and M. Wyart, Proc. Natl. Acad. Sci. 110, 6307 (2013).

[67] R. N. Widmer, G. I. Lampronti, S. Anzellini, R. Gaillac, S. Farsang, C. Zhou, A. M. Belenguer, C. W. Wilson, H. Palmer, A. K. Kleppe et al., Nat. Mater. 18, 370 (2019).

[68] S. Singh, M. D. Ediger, and J. J. De Pablo, Nat. Mater. 12, 139 (2013).

[69] S. Sawamura, R. Limbach, H. Behrens, and L. Wondraczek, J. Non-Cryst. Solids 481, 503 (2018).

[70] S. Sawamura and L. Wondraczek, Phys. Rev. Materials 2, 092601(R) (2018).

[71] R. Boehmer, K. L. Ngai, C. A. Angell, and D. J. Plazek, J. Chem. Phys. 99, 4201 (1993).

[72] M. L. F. Nascimento and C. Aparicio, J. Phys. Chem. Solids 68, 104 (2007).

[73] J. E. Shelby, J. Appl. Phys. 50, 8010 (1979).

[74] Y. Kawamura and A. Inoue, Appl. Phys. Lett. 77, 1114 (2000).

[75] T. D. Bennett, Y. Z. Yue, P. Li, A. Qiao, H. Z. Tao, N. G. Greaves, T. Richards, G. I. Lampronti, S. A. T. Redfern, F. Blanc et al., J. Am. Chem. Soc. 138, 3484 (2016).

[76] S. Li, R. Limbach, L. Longley, A. A. Shirzadi, J. C. Walmsley, D. N. Johnstone, P. A. Midgley, L. Wondraczek, and T. D. Bennett, J. Am. Chem. Soc. 141, 1027 (2019).

[77] M. Stepniewska, K. Januchta, S. Zhou, A. Qiao, M. M. Smedskjaer, and Y. Z. Yue, Proc. Natl. Acad. Sci. U.S.A. 117, 10149 (2020).

[78] I. Gallino, Entropy 19, 483 (2017).

[79] S. Scudino, B. Jerliu, K. Surreddi, U. Kühn, and J. Eckert, J. Alloys Compd. 509, S128 (2011).

[80] W. L. Johnson, J. H. Na, and M. D. Demetriou, Nat. Commun. 7, 10313 (2016).

[81] S. Wei, M. Stolpe, O. Gross, Z. Evenson, I. Gallino, W. Hembree, J. Bednarcik, J. J. Kruzic, and R. Busch, Appl. Phys. Lett. 106, 181901 (2015).

[82] G. N. de Macedo, S. Sawamura, and L. Wondraczek, J. NonCryst. Solids 492, 94 (2018).

[83] J. Deubener, H. Behrens, R. Müller, S. Zietka, and S. Reinsch, J. Non-Cryst. Solids 354, 4713 (2008).

[84] R. Limbach, A. Winterstein-Beckmann, J. Dellith, D. Möncke, and L. Wondraczek, J. Non-Cryst. Solids 417-418, 15 (2015).
[85] K. Griebenow, C. B. Bragatto, E. I. Kamitsos, and L. Wondraczek, J. Non-Cryst. Solids 481, 447 (2018).

[86] Q. H. Le, T. Palenta, O. Benzine, K. Griebenow, R. Limbach, E. I. Kamitsos, and L. Wondraczek, J. Non-Cryst. Solids 477, 58 (2017).

[87] Y.-F. Niu, J.-P. Guin, A. Abdelouas, T. Rouxel, and J. Troles, J. Non-Cryst. Solids 357, 932 (2011).

[88] F. Scarponi, L. Comez, D. Fioretto, and L. Palmieri, Phys. Rev. B 70, 054203 (2004).

[89] D. Moencke, E. I. Kamitsos, D. Palles, R. Limbach, A. Winterstein-Beckmann, T. Honma, Z. Yao, T. Rouxel, and L. Wondraczek, J. Chem. Phys. 145, 124501 (2016).

[90] J. Málek and J. Shánělová, J. Non-Cryst. Solids 351, 3458 (2005).

[91] R. Limbach, K. Kosiba, S. Pauly, U. Kühn, and L. Wondraczek, J. Non-Cryst. Solids 459, 130 (2017).

[92] W. H. Wang, Prog. Mater. Sci. 57, 487 (2012).

[93] S. Pauly, J. Das, N. Mattern, D. Kim, and J. Eckert, Intermetallics 17, 453 (2009).

[94] Y. Kawamura, T. Nakamura, H. Kato, H. Mano, and A. Inoue, Mater. Sci. Eng. A 304-306, 674 (2001), rQ10, Tenth International Conference on Rapidly Quenched and Metastable Materials.

[95] N. Nollmann, I. Binkowski, V. Schmidt, H. Rösner, and G. Wilde, Scr. Mater. 111, 119 (2016).

[96] E. Rössler, K.-U. Hess, and V. Novikov, J. Non-Cryst. Solids 223, 207 (1998).

[97] L. Muñoz-Senovilla and F. Muñoz, J. Non-Cryst. Solids 385, 9 (2014).

[98] S. Sirotkin, R. Meszaros, and L. Wondraczek, Int. J. Appl. Glass Sci. 3, 44 (2012).

[99] N. Da, S. Krolikowski, K. H. Nielsen, J. Kaschta, and L. Wondraczek, J. Am. Ceram. Soc. 93, 2171 (2010).

[100] G. Wilde, I. Lu, and R. Willnecker, Mater. Sci. Eng. A 375377, 417 (2004).

[101] E. Robin, J.-B. Le Cam, X. Balandraud, E. Toussaint, and L. Brilland, J. Non-Cryst. Solids 391, 101 (2014).

[102] P. Li, P. Gao, Y. Liu, and L.-M. Wang, J. Alloys Compd. 696, 754 (2017).

[103] M. A. Ramos, Philos. Mag. 84, 1313 (2004).

[104] C. Angell, J. Non-Cryst. Solids 131-133, 13 (1991), proceedings of the International Discussion Meeting on Relaxations in Complex Systems.

[105] L. Battezzati, Mater. Trans. 46, 2915 (2005).

[106] M. F. Ando, O. Benzine, Z. Pan, J.-L. Garden, K. Wondraczek, S. Grimm, K. Schuster, and L. Wondraczek, Sci. Rep. 8, 5394 (2018).

[107] B. Terziyska, I. Bivas, and K. Nenkov, Cryogenics 49, 171 (2009).

[108] Y. Li, P. Yu, and H. Bai, J. Appl. Phys. 104, 013520 (2008). 